\section{Le gros arbre qui cachait la forêt}

Florent Elefteriou, Patricia Ducy
Department of Molecular

and Human Genetics,

Baylor College of Medicine,

Houston, TX 77030,

États-Unis.

positif de l'absence de leptine sur la masse osseuse, capable même de contrebalancer la perte osseuse induite par une «ménopause constitutive », confère un attrait pharmaceutique certain à la recherche d'inhibiteurs de cette voie de signalisation, potentiellement efficaces pour traiter ou prévenir l'ostéoporose, qui accepterait d'être protégé de l'ostéoporose au prix d'une obésité! C'est en gardant à l'esprit ces deux aspects critiques de la fonction de la leptine que nous avons poursuivi nos travaux, qui viennent d'être récemment publiés [3].

Utilisant comme précédemment une approche combinant physiologie et génétique, nous avons d'abord démontré l'indépendance des signaux inhibiteurs relayant les fonctions anorexigène et anti-ostéogénique de la leptine (Tableau I). Deux composés chimiques, le monosodium glutamique (MSG) et le thioglucose d'or (GTG) sont connus pour engendrer des lésions neuronales distinctes. Exploitant cette particularité, nous avons analysé le poids et la masse osseuse de souris traitées par le MSG ou le $\mathrm{GTG}^{\star}$, certaines recevant simultanément une injection de leptine dans le troisième ventricule cérébral. Les souris traitées par le MSG étaient clairement obèses, mais conservaient une masse

\footnotetext{
* Une obésité acquise (par opposition aux obésités des modèles génétiques $o b / o b$ et $d b / d b$ ), peut être induite chez l'animal par deux produits: le monosodium glutamate (MSG) chez le nouveau-né, ou le gold thioglucose (GTG) chez l'adulte, qui tous deux induisent des lésions hypothalamiques. Dans le cas de l'« aurothioglucose », qui induit une obésité avec hyperphagie, le glucose permet au complexe orglucose de traverser la barrière ventromédiane de l'hypothalamus, région qui, normalement, inhibe la sensation de faim. La présence d'or est délétère et altère le fonctionnement normal des neurones sensibles au glucose de cette région.
}

Tableau I. Dissociation des activités anorexigène et anti-ostéogénique de la leptine. MSG :

monosodium glutamique; GTG: thioglucose d'or.

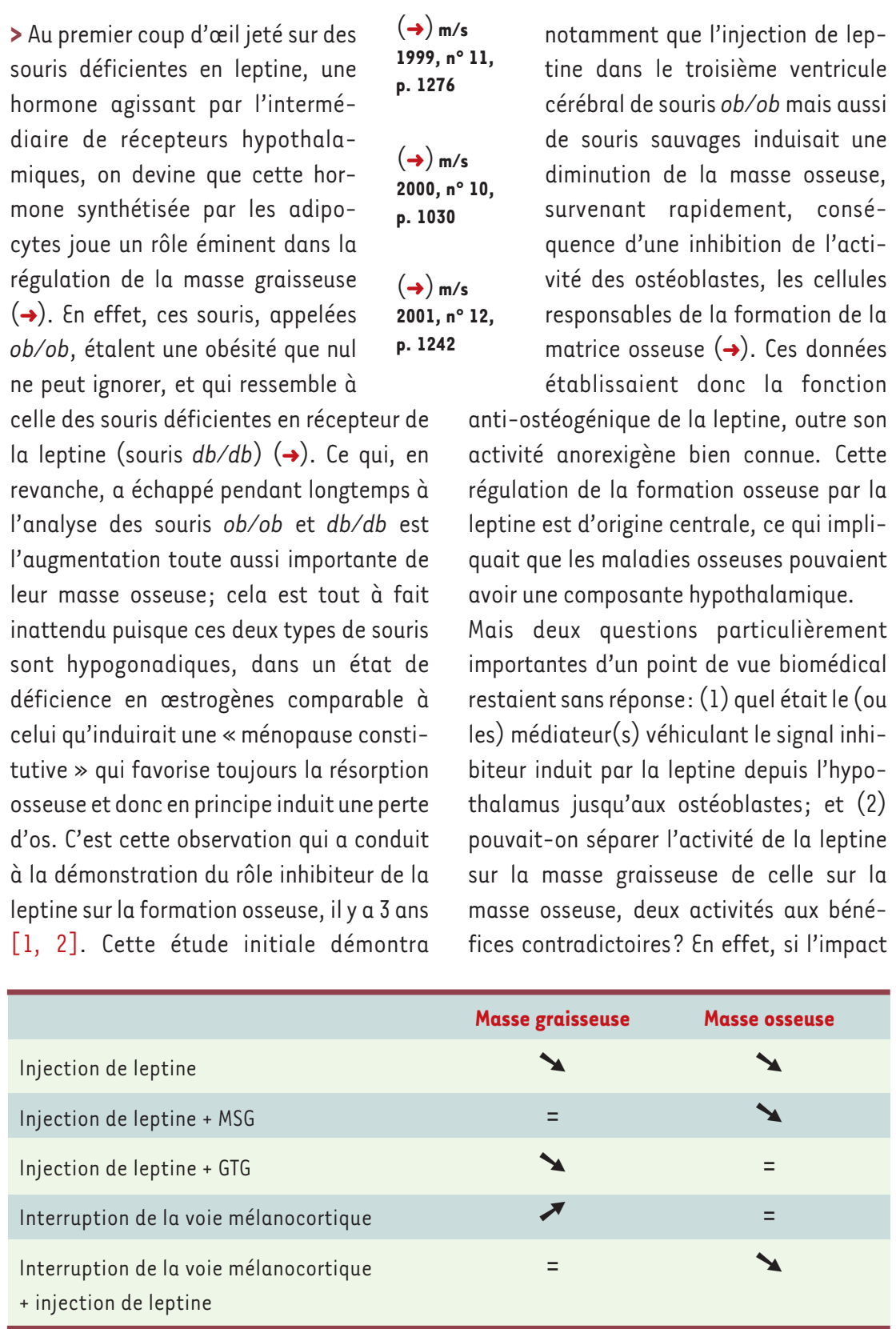


osseuse normale. Elles gardèrent la capacité de répondre à l'administration centrale de leptine par une inhibition de la formation osseuse, mais ne perdirent aucun poids. À l'inverse, la masse osseuse des souris traitées par le GTG augmentât, et cette augmentation ne fut pas corrigée par l'infusion centrale de leptine, alors que leur poids décrut. Ces deux expériences de physiologie établissaient l'indépendance des réseaux de neurones impliqués dans la régulation du poids et de la masse osseuse par la leptine. L'analyse de modèles murins, dans lesquels les signaux qui relayent l'activité anorexigène de la leptine sont bloqués, confirma cette indépendance au niveau génétique. En effet, la masse osseuse n'est pas augmentée chez les souris déficientes en CART (cocaine amphétamine related transcript), un neuropeptide transmettant le signal anorexigène de la leptine [4], et l'inactivation de la voie de signalisation mélanocortique, une cause d'obésité chez l'homme comme chez la souris [5, 6], n'entraine pas non plus d'accroissement de la masse osseuse. De plus, alors que ce blocage provoque une désensibilisation à l'action de la leptine sur la masse graisseuse [7], l'effet sur la perte osseuse de l'hormone injectée par voie intra-ventriculaire est comparable à celui qui est observé chez des souris sauvages. Ayant démontré l'indépendance des médiateurs anorexigènes et anti-ostéogéniques contrôlés par la leptine, nous avons ensuite entrepris de les identifier. Leur transmission par voie nerveuse plutôt qu'humorale fut établie par une expérience de parabiose. La circulation sanguine de deux souris $o b / o b$ fut couplée chirurgicalement et l'une d'entre elles reçut de la leptine par voie centrale intra-ventriculaire. Seule cette dernière diminua sa masse osseuse, l'autre non, établissant que le signal inhibant la formation osseuse et engendré par la leptine n'était pas transmis d'une souris à l'autre par l'intermédiaire de la circulation sanguine. Cette expérience pointait une transmission neuronale. Considérant que les souris ob/ob ont une diminution de l'activité sympathique [8], cette composante du système nerveux autonome s'imposait comme candidate. Le système nerveux sympathique (SNS) véhicule principalement des informations adaptatives réflexes jusqu'aux muscles et aux principaux organes, contrôlant par exemple la fréquence cardiaque. L'information sympathique est transmise par la sécrétion au niveau des terminaisons nerveuses de catécholamines (épinéphrine et norépinéphrine) qui se lient à des récepteurs adrénergiques ( $\alpha$ ou $\beta$ ) présents sur les cellules effectrices des organes cibles. Pour démontrer le rôle du SNS dans le contrôle de la formation osseuse par la leptine, nous avons initialement analysé les os de souris déficientes en DBH (dopamine $\beta$-hydroxylase), l'enzyme nécessaire à la production des catécholamines. Ces souris montrant une augmentation de la masse osseuse - augmentation qui plus est insensible à l'injection cérébrale de leptine - la fonction médiatrice du SNS dans l'activité inhibitrice de la leptine sur l'os devint évidente. Des expériences complémentaires démontrèrent que les ostéoblastes expriment des récepteurs adrénergiques $\beta 2$ fonctionnels qui répondent à des traitements par la norépinéphrine ou l'isoprotérénol - un agoniste des récepteurs $\beta$-adrénergiques - précisant le mécanisme de la connexion système nerveux périphériqueformation osseuse. Le traitement de souris par ce même agoniste induit d'ailleurs une très forte inhibition de la formation osseuse, preuve que ce mécanisme intervient in vivo.

L'ensemble de cette étude proposait donc un schéma du mécanisme d'action de la leptine sur la formation osseuse, dont l'importance biomédicale fut établie par une expérience supplémen- taire. L'une des fonctions du SNS est de contrôler la pression artérielle, et il y a maintenant plusieurs décennies que furent développés, dans un but thérapeutique, des antagonistes des récepteurs adrénergiques regroupés sous le terme générique de « $\beta$-bloquants ». On pouvait donc supposer que la régulation de la formation osseuse par le SNS puisse, elle aussi, servir de base thérapeutique pour traiter les maladies osseuses du type de l'ostéoporose. Afin de valider cette hypothèse, des souris sauvages, ayant subi une ménopause chirugicale par ablation des ovaires, furent traitées avec du propanolol, un $\beta$ bloquant à large spectre. Conformément à la prédiction du modèle que nous avions établi, les ostéoblastes de ces souris, déréprimés par le blocage du signal négatif

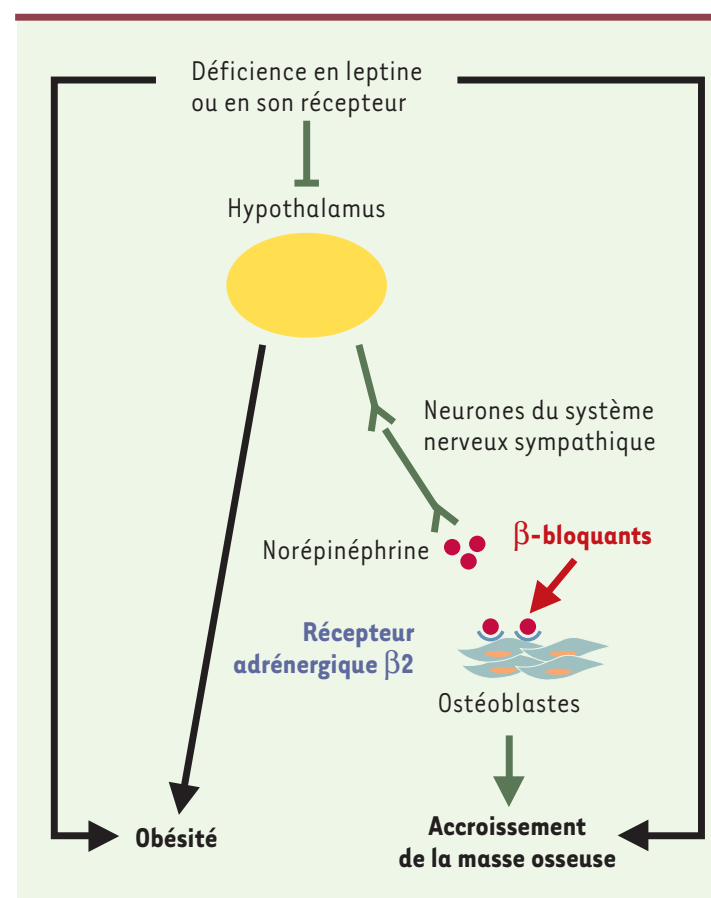

Figure 1. Mécanisme d'action de la leptine et des $\beta$-bloquants sur la masse osseuse. La leptine se lie à son récepteur au niveau de neurones hypothalamiques. Ce signal est véhiculé par le système nerveux sympathique jusqu'à l'os où les terminaisons nerveuses libèrent de la norépinephrine. Celle-ci se lie aux récepteurs adrénergiques exprimés par les ostéoblastes qui diminuent leur synthèse de matrice osseuse. L'absence de signalisation par la leptine au niveau de l'hypothalamus induit une obésité et un accroissement de la masse osseuse. En revanche, le blocage de cette voie de signalisation par administration de $\beta$-bloquants induit une augmentation de la masse osseuse mais n'affecte pas la masse graisseuse. 
provenant du SNS, augmentèrent leur production d'os. L'élément important est que la masse osseuse de ces souris est restée intacte alors que celle des souris non traitées par le propanolol décroissait.

En résumé, ces études ont montré que la leptine inhibe la formation osseuse par l'intermédiaire du SNS, et que le blocage de cette régulation peut être utilisé pour contrebalancer une perte osseuse pathologique (Figure 1). Cette situation prévaut chez la souris. Mais qu'en est-il chez l'homme? Pour l'instant, et malgré leur utilisation fréquente et déjà ancienne, il n'existe pas de données cliniques démontrant un effet positif des $\beta$-bloquants sur la masse osseuse. Cette constatation ne va cependant pas à l'encontre de notre modèle car, dans le domaine scientifique, l'absence de résultat n'est pas une preuve; des études rétrospectives ou prospectives pourront seules apporter une réponse claire dans le futur. II existe cependant toute une série d'arguments indirects qui d'ores et déjà suggèrent que nos résultats seront transposables à l'homme. Premièrement, il n'existe pas d'exemple où une protéine, qui est présente chez la souris et chez l'homme, assure une fonction physiologique majeure différente dans les deux espèces. Deuxièmement, les autres fonctions de la leptine sont conservées chez les mammifères. Troisièmement, le traitement par les $\beta$-bloquants est depuis longtemps connu comme le mode d'intervention le plus efficace pour soigner une maladie humaine appelée algoneurodystrophie dont l'un des symptômes majeurs est... une ostéoporose. $\diamond$

Control of bone mass by leptin occurs through

the sympathetic nervous system

\section{RÉFÉRENCES}

1. Ducy P, Amling M, Takeda S, et al. Leptin inhibits bone formation through a hypothalamic relay: $a$ central control of bone mass. Cell 2000; 100 : 197-207.

2. Karsenty G, Ducy P. Contrôle de la formation osseuse par l'axe hypothalamique: une nouvelle fonction de la leptine. Med Sci 2000; 16: 289-90.
3. Takeda S, Elefteriou F, Levasseur R, et al. Leptin regulates bone formation via the sympathetic nervous system. Cell 2002; 111: 305-17.

4. Kristensen $P$, Judge ME, Thim $L$, et al. Hypothalamic CART is a new anorectic peptide regulated by leptin. Nature 1998; 393: 72-6.

5. Huszar D, Lynch CA, Fairchild-Huntress $V$, et al. Targeted disruption of the melanocortin-4 receptor results in obesity in mice. Cell 1997; 88: 131-41.

6. Vaisse C, Clement K, GuyGrand B, Froguel P. A frameshift mutation in human MC4R is associated with a dominant form of obesity. Nat Genet 1998; 20: 113-4.

7. Halaas J L, Boozer C, BlairWest J, et al. Physiological response to long-term peripheral and central leptin infusion in lean and obese mice. Proc Natl Acad Sci USA 1997; 94 : 8878-83.

8. Bray GA, York DA. The MONA LISA hypothesis in the time of leptin. Recent Prog Horm Res 1998; 53: 95-117.

\section{NOUVELLE}

\section{Fentes et brides : une même étiologie pour deux syndromes a priori bien différents}

Simone Gilgenkrantz

labiale - mais jamais l'inverse - et de pouvoir affirmer son caractère autosomique dominant (pénétrance de

9, rue Basse, 54330 Clerey-sur-Brenon, France.

s.gilgenkrantz@chu-nancy.fr $96,7 \%$ ). Les analyses de ségrégation familiale ont montré que le locus se situe en $1 q 42-q 41 \quad$ [2]. La recherche du gène fut ren-

> Les gènes IRF (interferon regulatory factor), au nombre de 9 , codent pour une famille de facteurs de transcription qui contrôlent l'expression de l'interféron après une infection virale. Mais il est probable qu'ils interviennent aussi dans bien d'autres domaines, en particulier dans le contrôle du développement embryonnaire, comme en atteste la récente implication d'IRF6 dans deux maladies à transmission autosomique dominante: le syndrome de van der Woude et le syndrome du pterygium poplité [1].

\section{Le syndrome de van der Woude (VWS)}

Parmi les syndromes associés à des fentes labio-palatines, le VWS a été très tôt individualisé car il est caractérisé par la présence de deux petits pertuis symétriques au niveau de la lèvre inférieure (Figure 1A) qui permettent d'identifier cette variété de fente palatine pouvant s'accompagner de fente due difficile par la présence de nombreux polymorphismes (single nucleotide polymorphism) à ce locus (environ 1 pour 1900 $\mathrm{pb}$ ). C'est finalement grâce à une paire de jumeaux monozygotes discordants pour le VWS que la preuve fut faite de l'implication d'IRF6 dans la maladie [1]. En effet, seul le jumeau atteint était porteur d'une mutation non-sens dans l'exon 4, absente chez le jumeau sain et chez les parents. Par la suite, l'équipe ayant fait cette constatation a 